\title{
Perforation of Terminal Ileum as a Rare Complication of an Early Post- Transplant Course
}

\author{
Ivana Dedinská1, L'udovít Laca', Michal Kalman², Miroslav Pindura', Juraj Miklušica ${ }^{1 *}$, Blažej Palkoci', Denisa Osinová3, Peter Galajda ${ }^{4}$ and \\ Marián Mokáń ${ }^{4}$
}

${ }^{1}$ Surgery Clinic and Transplant Center, University Hospital Martin and Jessenius Faculty of Medicine, Comenius University, Slovakia ${ }^{2}$ Department of Pathology, University Hospital Martin and Jessenius Faculty of Medicine, Comenius University, Slovakia

${ }^{3}$ Clinic of Anesthesiology and Intenstive Medicine, University Hospital Martin and Jessenius Faculty of Medicine, Comenius University, Slovakia

${ }^{4}$ I. Internal Clinic University Hospital Martin and Jessenius Faculty of Medicine, Comenius University, Slovakia

\begin{abstract}
Perforations of any part of the gastrointestinal tract can occur in transplant patients, although the colon may be the most common site. We analyze the case of a 39-year-old patient after kidney transplantation who suffered perforation of terminal ileum with peritonitis on the 7th day after transplantation. We histologically confirmed presence of a foreign body in the intestinal wall - a rare cause of intestinal perforation in a patient after kidney transplantation. We have not noticed a significant worsening of graft function or development of sepsis mainly because of quick evaluation of the patient's clinical state, prompt surgical intervention and a combined antibiotic treatment. Gastrointestinal perforations have rarely been noted in large studies involving transplant recipients.
\end{abstract}

Keywords: Kidney transplantation; Intestinal perforation; Antibiotic treatment; Immunosupression

\section{Introduction}

Organ transplantation has emerged as the preferred treatment modality for end-stage liver, kidney, heart and lung diseases. The gastrointestinal tract accounts for a large component of nonallograft-related complications seen after all types of solid organ transplantationand is responsible for considerable morbidity and mortalityassociated with transplantation [1-4].

Upper bowel perforation can be described as either free or contained. Free perforation occurs when bowel contents spill freely into the abdominal cavity, causing diffuse peritonitis (eg, duodenal or gastric perforation). Contained perforation occurs when a fullthickness hole is created by an ulcer, but free spillage is prevented because contiguous organs wall off the area (as occurs, for example, when a duodenal ulcer penetrates into the pancreas). Lower bowel results in free intraperitoneal contamination [5].

Thompson et al. first described ileocolonic perforation in patients after kidney transplantation in 1975. Out of 248 patients after kidney transplantation, eleven patients developed ileocolonic perforation. Only three of the patients survived this complication and in each case the perforation was localized [6]. Perforations of any part of the gastrointestinal tract can occur in transplant patients, although the colon may be the most common site. Perforation occurs most commonly in patients with renal failure who are undergoing dialysis and have had heavy immunosuppression, particularly with corticosteroids. Early perforations are considered to be largely attributable to diverticulitis or CMV colitis [7].

Symptoms of gastrointestinal perforation may include severe stomach pain, chills, fever, nausea and vomiting. When peritonitis occurs, the abdomen feels very tender. In addition to the general symptoms of perforation, symptoms of peritonitis may include exhaustion, shortness of breath or tachycardia.

The goals of surgical therapy are as follows:

-To correct the underlying anatomical problem

-To correct the cause of peritonitis

-To remove any foreign material in the peritoneal cavity that might inhibit WBC function and promote bacterial growth (eg, feces, food, bile, gastric or intestinal secretions, blood) [5].

-However, if symptoms and signs of generalized peritonitis are absent, a nonoperative policy may be used with antibiotic therapy directed against gram-negative and anaerobic bacteria.

The causes of intestinal perforation in patients following organ transplantation are well known and are mainly related to administered immunosuppression after transplantation. In our case report which describe terms of intestinal perforation in a short post-transplant period, where the foreign body causing perforation.

\section{Case Presentation}

In the set of patients who underwent kidney transplantation in Transplant Center Martin from 2003 to $2015(n=250)$ we identified only one patient who had developed perforation of the gastrointestinal tract in the post-transplant period.

We present the casuistics of a 39-year-old man patient who underwent primary kidney transplantation from a living donor (58-year-old mother) in $04 / 2015$. The recipient was in a regular hemodialysis program from $03 / 2015$ and the cause of native kidney failure was a suspected chronic glomerulonephritis of the solitary kidney (biopsy of the native kidney was not carried out). The patient was prepared for transplantation already in pre-dialysis period, so the patient could undergo a transplant very short time (1 month) after initiation of dialysis program. Cross match test (by using flow cytometry) before transplantation was 2 times negative and PRA were $0 \%$. In case of induction we chose basiliximab due to the

*Corresponding author: Juraj Miklušica, Surgery Clinic and Transplant Center University Hospital Martin and Jessenius Faculty of Medicine, Comenius University, Slovakia, Tel: + 421 434203246, e-mail: juraj.miklusica@gmail.com

Received September 03, 2015; Accepted November 02, 2015; Published November 09, 2015

Citation: Dedinská I, Laca L, Kalman M, Pindura M, Miklušica J, et al. (2015) Perforation of Terminal lleum as a Rare Complication of an Early Post-Transplant Course. J Clin Case Rep 5: 631. doi:10.4172/2165-7920.1000631

Copyright: (c) 2015 Dedinská I, et al. This is an open-access article distributed under the terms of the Creative Commons Attribution License, which permits unrestricted use, distribution, and reproduction in any medium, provided the original author and source are credited. 
low immunological risk of the recipient ( 1 mismatch in HLA A) in combination with methyl-prednisolone (500 $\mathrm{mg}$ intravenous on D0 and D1), immunosuppressive regimen consisted of tacrolimus (target level $5-10 \mathrm{ng} / \mathrm{ml}$ ), everolimus (target level $3-8 \mathrm{ng} / \mathrm{ml}$ ) and $20 \mathrm{mg}$ prednisone/day (regimen was adjusted according to the protocol of the clinical study in which the patient is engaged). After transplantation we noticed only small decrease in creatinine and diuresis was around the level of residual diuresis. On the second post-surgery day we have proven positivity in B-lymphocyte cross-match test (it is highly unlikely that cross match after transplantation was a false positive, donor specific antibodies by using flowPRA were negative, so we supposed an nonHLA acute antibody-mediated rejection) and consequently realized 3 high-volume plasmafereses with $5 \%$ albumin in combination with intravenous immunoglobulins (total dose 94.5 grams). Diuresis

had developed after this treatment, and a decrease in nitrogenous substances was noticed as well. The check cross-match test on the 6th post-surgery day was negative.

On the 7th day after surgery an acute abdomen pain and vomiting appears, accompanied by tachycardia, hypotensia and shiver. An x-ray image of the abdomen was urgently made and pneumoperitonea was diagnosed. The patient was immediately taken to the operating theatre. During the examination of abdominal cavity perforation of terminal ileum with peritonitis was discovered. During the surgical procedure was realized wash-out of the abdominal cavity and end to end anastomosis of ileum. There was just one drain placed and we removed it after 2 days.

We have also laboratory recorded a significant rise in inflammatory parameters and a slight deterioration of graft function (Graph 1). We have confirmed presence of Klebsiella pneumoniae and Enterococcus faecium by cultivation (sensitivity to antibiotics is shown (Tables 1 and 2). We immediately started with combined antibiotic treatment (meropenem - reduced dose according to the actual glomerular filtration, linezolid $600 \mathrm{mg}$ intravenous $2 \mathrm{x}$ per day and natamycin per os $3 x$ per day). Three fragments of the perforated part of the intestine were histologically analyzed and a complex of reactive changes such as vascular congestion, bleeding and coagulative necrosis was discovered, inside we found remains of foreign material accompanied by a purulent inflammation without signs of a neoplastic process (Figure 1). We have not proven CMV infection in the patient (CMV viremia established by
PCR was negative at the time of perforation).

Further post-surgery course was not complicated. The antibiotic treatment lasted 10 days and immunosuppression was not interrupted or reduced. The patient is currently in an excellent condition without any problems. After completion of history regarding foreign body as a cause perforation of the ileum, we can assume that it could be the nucleolus of apples or cherries.

\section{Discussion}

Perforation of the colon is the most feared consequence of these complications, because the mortality rises to over 50\% in transplanted group of patients [8]. A delay in the diagnosis in patients after transplantation is common, as the symptoms and signs are sparse and often masked by immunosuppression [9]. The incidence of colon perforations is $1-2 \%$ in kidney transplantation patients $[10,11]$. Diverticulitis is the most common cause $[9,12]$. Other causes can be ischaemia or CMV colitis [12].

Large, prospective, randomized trials with gastrointestinal complications or intestinal perforation as the primary endpoint do not exist and data found in literature are only at the level of case reports.

Authors retrospectively evaluated gastrointestinal complications in more than 1500 patients. Out of this number of patients, only 8 of them suffered intestinal perforation after transplantation $(0.53 \%)$.

Half the perforations were caused by diverticulitis. Although they were few in number, $25 \%$ of these complications were fatal. The diagnosis was often delayed because immunosuppressive drugs might have masked symptoms and affected the patients' responses to the septic condition. All fatal colon perforations in these series occurred during the first year after the transplantation [1].

Similarly, in the study of authors Catena et al., perforation of gastrointestinal tract was a very serious complication of the posttransplant course with high mortality. This study reports major gastrointestinal complications among a group of 1611 patients following kidney transplantation. The immunosuppressive regimen changed somewhat during the course of the study but included azathioprine, prednisolone, antilymphocyte globulin, cyclosporine, tacrolimus, mycophenolate mofetil, and sirolimus. Perforations occurred in the colon $(n=21)$, small bowel $(n=15)$, duodenum $(n=6)$,

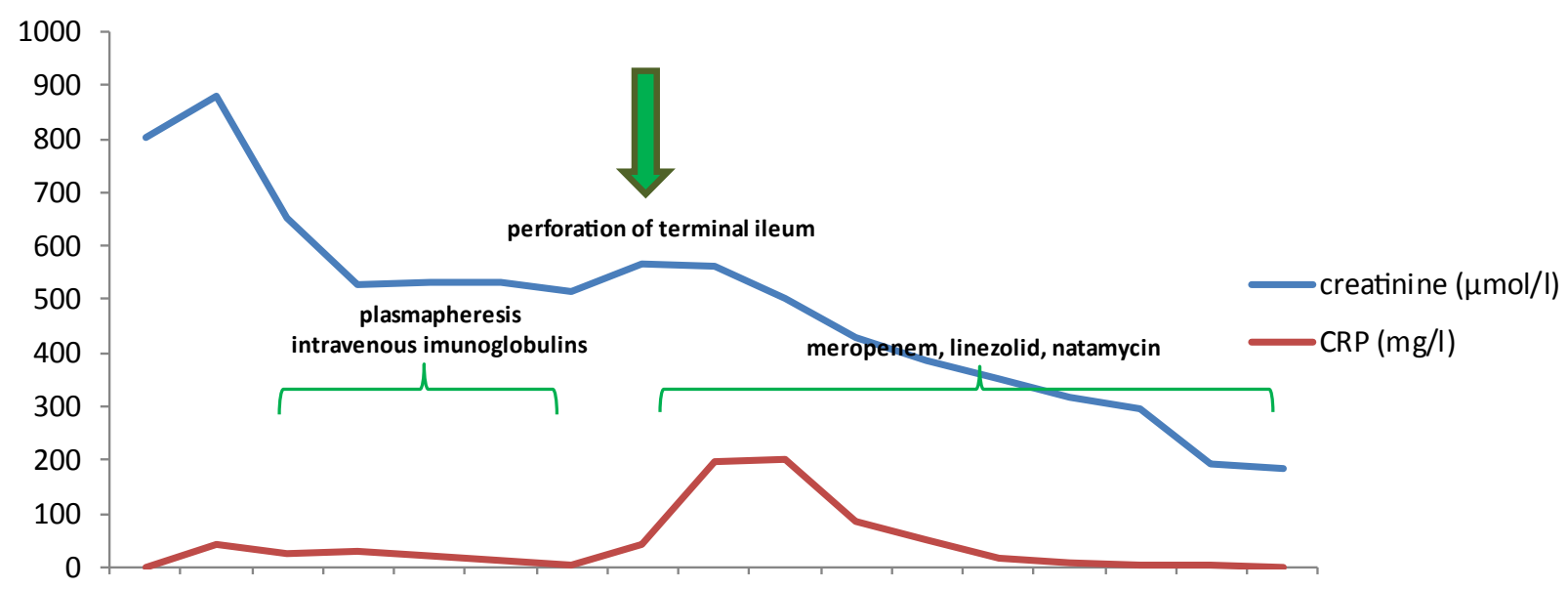

day 0 day 1 day 2 day 3 day 4 day 5 day 6 day 7 day 8 day 9 day 10 day 11 day 12 day 13 day 14 day 15 day 16

Graph 1: Levels of creatinine and C-reactive protein in the post-transplant period. 


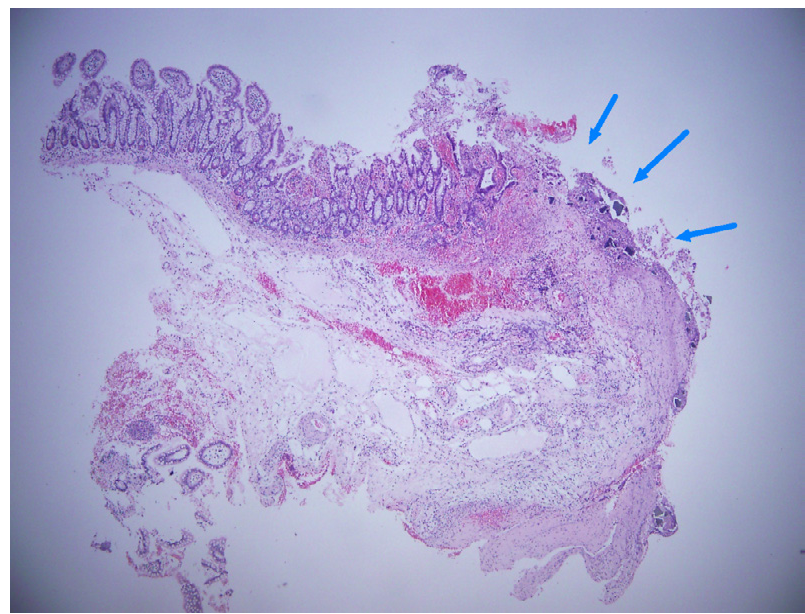

Figure 1: Hematoxylin and Eosin shows small intestine specimen with perforation and basophilic crystals in the fibrinopurulent material (blue arrows).

\begin{tabular}{|l|l|l|l|}
\hline Amikacin & Sensitive & Amoxicillin+Clavulanate & Resistant \\
\hline Ampicilin & Resistant & Aztreonam & Resistant \\
\hline Cefalexin & Resistant & Cefepime & Resistant \\
\hline $\begin{array}{l}\text { Cefoperazone }+ \\
\text { Sulbactam }\end{array}$ & Sensitive & Cefotaxime & Resistant \\
\hline Cefoxitim & Sensitive & Ceftazidime & Resistant \\
\hline Ciprofloxacin & Resistant & Ertapenem & Sensitive \\
\hline Levofloxacin & Resistant & Imipenem & Sensitive \\
\hline $\begin{array}{l}\text { Piperacillin+ } \\
\text { Tazobactam }\end{array}$ & Intermediarysensitive & Meropenem & Sensitive \\
\hline
\end{tabular}

Table 1: Sensitivity to antibiotics Klebsiella pneumoniae -cultivation from the wound after perforation of terminal ileum.

\begin{tabular}{|l|l|l|l|}
\hline Amoxicillin+Clavulanate & Resistant & Vancomycin & Sensitive \\
\hline Cefepime & Resistant & Ampicillin & Resistant \\
\hline Cefotaxime & Resistant & Cefoperazone+Sulbactam & Resistant \\
\hline Ciprofloxacin & Resistant & Ceftazidime & Resistant \\
\hline Gentamicin & Resistant & Ertapenem & Resistant \\
\hline Imipenem & Resistant & Chloramphenicol & Sensitive \\
\hline Meropenem & Resistant & Linezolid & Sensitive \\
\hline Teicoplanin & Sensitive & Piperacillin+ Tazobactam & Resistant \\
\hline & & Tetracycline & Resistant \\
\hline
\end{tabular}

Table 2: Sensitivity to antibiotics Enterococcus faecium - cultivation from the wound after perforation of terminal ileum.

and stomach $(\mathrm{n}=4)$. Nearly $50 \%$ of the complications occurred while patients were being given high-dose immunosuppression to manage either the early post-operative period or acute rejection episodes. Of the 46 patients affected, 11 (24\%) died as a direct result of the gastrointestinal complications. This high mortality appeared to be related to the effects of the immunosuppression and the associated response to sepsis [13].

In case of our described case we excluded diverticulosis and CMV infection from the possible risk factors for perforation of the intestine after kidney transplantation (by inspection during operation). Perforation might have been caused by the immunosuppressive therapy; however, we have rarely diagnosed presence of a foreign body (probably a seed, or a pip) by a histological examination.

Due to the prompt diagnostics and a quick surgical intervention the patient had not developed sepsis in the immunosuppressive environment. The patient was afebrile and with a targeted antibiotic treatment a prompt decrease in inflammatory parameters was recorded. During the post-transplant period it was not necessary to discontinue or reduce the immunosuppressive therapy (per os intake was maintained due to localization of the perforation), this made it possible to maintain stabilized graft function, moreover, we later recorded a decrease in the creatinine level.

\section{Conclusion}

Perforation of intestine in a patient after kidney transplantation is a serious complication with high mortality. Perforation of the intestine caused by presence of a foreign body is not currently mentioned in any literature, it is considered to be a very rare complication. In case of development of perforation of the intestine during the post-transplant period a prompt diagnosis and a quick surgical solution are important, as well as a targeted combined antibiotic treatment.

\section{References}

1. Sarkio S, Halme L, Kyllonen L, Salrnela K (2004) Severe gastrointestinal complications after ,515 adult kidney transplantations. Transpl Int 17: 505-510.

2. Hoekstra HJ, Hawkins K, Boer WJ (2001) Gastrointestinal complications in lung transplant survivors that require surgical intervention. Br J Surg 88: 433-438.

3. Luckraz H, Goddard M, Charman SC, Wallwork J, Parameshwar J, et al. (2005) Early mortality after cardiac transplantation: should we do better? J Heart Lung Transplant 24: 401-405.

4. Goldberg HJ, Hertz MI, Ricciardi R (2006) Colon and rectal complications after heart and lung transplantation. J Am Coll Surg 202: 55-61.

5. Azer AS, Takavera F, Geibel J, Grosso MA. Intestinal Perforation. Medscape 2014.

6. Thompson WM, Seigler HF, Rice RP (1975) Ileocolonic perforation a complication following renal transplantation. American Journal of Roentgenology 125: 723-730.

7. Helderman JH, Goral S (2002) Gastrointestinal complications of transplant immunosuppression. J Am Soc Nephrol 13: 277-287.

8. Benoit G, Moukarzel M, Verdelli G, Hiesse C, Buffet C, et al. (1993) Gastrointestinal complications in renal transplantation. Transpl Int 6: 45-49.

9. Dominquez Fernandez E, Albrecht K, Heeman U (1998) Prevalence of diverticulosis and incidence of bowel perforation after kidney transplantation in patients with polycystic kidney disease. Transpl Int 11: 28-31.

10. Church JM, Fazio VW, Braun WE, Novick AC, Steinmuller DR (1986) Perforation of the colon in renal homograft recipients. A report of 11 cases and a review of the literature. Ann Surg 203: 69-76.

11. Stelzner M, Vlahakos DV, Milford EL, Tilney NL (1997) Colonic perforations after renal transplantation. J Am Coll Surg 184: 63-69.

12. Remzi FH (2002) Colonic complications of organ transplantation. Transplant Proc 34: 2119-2121.

13. Catena F, Ansaloni L, Gazzotti F (2008) Gastrointestinal perforations following kidney transplantation. Transplant Proc 40: 1895-1896. 